\title{
Primary Resistance to Brigatinib in a Patient with Lung Adenocarcinoma Harboring ALK G I202R Mutation and LIPI-NTRKI Rearrangement
}

This article was published in the following Dove Press journal: OncoTargets and Therapy

\author{
Zhiwei Xiao' ${ }^{1, *}$ \\ Xuewu Huang ${ }^{1}, *$ \\ Biyuan $\mathrm{Xie}^{2}$ \\ Wenzhuan Xie $\mathbb{1 D}^{3}$ \\ Mengli Huang (iD ${ }^{3}$ \\ Lizhu Lin $\mathbb{D}^{\prime}$ \\ 'Oncology Center, The First Affiliated \\ Hospital of Guangzhou University of \\ Chinese Medicine, Guangzhou 510405, \\ Guangdong, People's Republic of China; \\ ${ }^{2}$ Oncology Department, The Second \\ Traditional Chinese Medicine Hospital of \\ Guangdong Province, Guangzhou \\ 510405, Guangdong, People's Republic of \\ China; ${ }^{3}$ The Medical Department, 3D \\ Medicines Inc, Shanghai 20III4, People's \\ Republic of China \\ *These authors contributed equally to \\ this work
}

Purpose: Anaplastic lymphoma kinase $(A L K)$ inhibitors have transformed the management of non-small-cell lung cancer (NSCLC) patients with $A L K$ gene rearrangement. This paper reports a new resistance mechanism to a second-generation ALK inhibitor, brigatinib.

Case Report: A 43-year-old woman who had no history of smoking was diagnosed with stage IVa (T2bN2M1b) lung adenocarcinoma. After the first-line chemotherapy failed, the patient received crizotinib due to the presence of $E M L 4-A L K$ fusion by next-generation sequencing (NGS). The patient had disease progression after 8 months on crizotinib, and a second NGS identified the $A L K$ G1202R resistance mutation. Therefore, she was switched to brigatinib. After only 53 days of treatment with brigatinib, the patient developed a new $1.6 \times 1.2 \mathrm{~cm}$ lesion in the mediastinal lymph node. A third NGS testing revealed a new form of NTRK rearrangement (LIPI-NTRK1). The patient died 16 months after diagnosis.

Conclusion: This paper provides new insights into the primary resistance to brigatinib in NSCLC patients carrying $A L K$ G1202R mutation. The new fusion form of NTRK rearrangement was detected, which may provide potential treatment options after brigatinib resistance.

Keywords: NTRK1, ALK, primary resistance, brigatinib, NSCLC

\section{Introduction}

Anaplastic lymphoma kinase $(A L K)$ rearrangements are present in approximately 5\% of non-small-cell lung cancer (NSCLC) patients, defining a specific molecular subgroup. ${ }^{1}$ The $A L K$ gene copy number gain and amplification (ALK-A) are well studied in different types of cancer, and could affect the rearrange of the $A L K$ gene in NSCLC. ${ }^{2}$ Crizotinib is effective as the first-in-class inhibitor in $A L K$-positive NSCLC, ${ }^{3}$ but most patients acquire resistance against ALK inhibitors through different molecular mechanisms, ${ }^{4}$ including the co-occurrence of $A L K-\mathrm{A}$ and $A L K$ rearrangement. ${ }^{2}$ Brigatinib is a next-generation oral ALK inhibitor to treat metastatic $A L K+$ NSCLC patients who have progressed on or are intolerant of crizotinib. ${ }^{5}$ Efficacy data confirmed that brigatinib is an effective therapeutic strategy after crizotinib failure. ${ }^{5,6}$ Previous reports showed that brigatinib can overcome resistance to first- and secondgeneration ALK inhibitors caused by secondary mutations such as $A L K$ G1202R. ${ }^{7,8}$ According to the preliminary data of the Phase 2 ALTA trial, the investigator-assessed median progression-free survival (PFS) was 12.9 months in patients treated with brigatinib. ${ }^{9}$ However, there are some conflicting views on the resistance mechanisms
Correspondence: Lizhu Lin Email lizhulin26@yahoo.com 
of brigatinib. Sharma et al reported that the G1202R mutation might cause acquired resistance to brigatinib. This may result from the steric clash between the side chain of G1202R and the extended solubilization group of brigatinib. ${ }^{10}$

Chromosomal rearrangements involving neurotrophic tyrosine kinase 1 (NTRK1) occur at an extremely low frequency of approximately $0.1 \%$ to $1 \%$ in NSCLC and they are highly therapeutically actionable drivers of tumor growth. ${ }^{11,12}$ Entrectinib is an orally available small molecule for the treatment of various solid tumors harboring NTRK1/NTRK2/ NTRK3 or $A L K$ gene fusions. ${ }^{13}$ Despite durable responses to TRK-directed therapy in patients with NTRK-rearranged tumors, it is expected that most patients eventually develop acquired resistance. Moreover, NTRK-rearranged NSCLCs seem to be oncogene-dependent and not combined with $A L K$ or ROS1 gene rearrangements. ${ }^{14,15}$

We here reported a case of lung adenocarcinoma carrying the G1202R ALK mutation and a new oncogenic NTRK fusion variant who was resistant to brigatinib treatment.

\section{Case Presentation}

A 43-year-old female never-smoker presented with prolonged paroxysmal cough and was diagnosed with stage IVa (T2bN2M1b) lung adenocarcinoma in Jun 2017. She underwent an endobronchial ultrasound-guided transbronchial needle aspiration (EBUS-TBNA) and immunohistochemistry showed that the nucleoli had obvious heterotypic cells arranged like adenoid and CK (+), TTF-1 (+), NapsinA (+), P63 (+). Enhanced computed tomography (CT) revealed an upper left lung lesion $(3.6 \times 2.9 \mathrm{~cm})$ with mediastinal lymph node metastasis $(2.1 \times 1.0 \mathrm{~cm})$ and hepatic $\mathrm{S} 4$ segment metastasis $(1.0 \times 0.9 \mathrm{~cm})$. She received two courses of docetaxel combined with cisplatin chemotherapy as the first-line treatment, and CT revealed no change in the lesion (Figure 1). To explore potential targeted treatment, next-generation sequencing (NGS) analysis was performed on the patient's peripheral blood using a 21 gene panel. The patient was found to carry the classical EML4-ALK fusion. Therefore, crizotinib was commenced at $250 \mathrm{mg}$ bid on September 5th 2017. A followup CT conducted on January 17th, 2018 revealed a $61 \%$ regression in her primary lung lesion $(1.4 \times 1.2 \mathrm{~cm})$, indicating that the patient had achieved partial response (PR). In May 2018, eight months after the onset of crizotinib treatment, the patient was discovered to have tumor progression (PD) due to brain metastases $(1.9 \times 1.6 \mathrm{~cm})$ by head MRI and acquired resistance to crizotinib was suspected.

A second blood-based NGS showed the presence of the $p$. G1202R ALK mutation was observed. The patient was started on brigatinib (180 mg daily with a seven-day lead-in at $90 \mathrm{mg}$ ) on May $18^{\text {th }}, 2018$. Brigatinib is a next-generation oral ALK inhibitor used in the second-line after progression on crizotinib. However, a CT scan conducted after 53 days of brigatinib treatment revealed a new mediastinal lymph node $(1.6 \times 1.2 \mathrm{~cm})$, and the appearance of new pericardial metastases. A third NGS testing was therefore performed, and a new type of NTRK arrangement (LIPI-NTRK1, Figure 2) was identified in addition to the two previous alternations. The patient's shortness of breath significantly increased due to the hydrothorax on the left side of the chest. After a pleural puncture, the symptoms were slightly relieved. Given her physical conditions, the patient refused to switch to another regimen, including NTRK inhibitors. She was able to benefit from ALK TKI therapy for 8 months and died on September $24^{\text {th }}, 2018$, with overall survival of 16 months from the time of diagnosis.

\section{Discussion}

In this case report, a new form of NTRK rearrangement (LIPI-NTRK1) was identified, which included inversion of LIPI exon 1 and NTRK1 exons 8-17. In addition, the classical $E M L 4-A L K$ fusion and the $A L K$ G1202R primary resistance mutation were also detected. Despite multiple lines of targeted treatment guided by NGS testing, this patient failed to benefit from the treatment of brigatinib due to the emergence of resistance mutations.

ALK-TKIs are widely used in clinical practice, but patients' responses are heterogeneous due to the emergence of resistance genes. Numerous researchers have explored the mechanisms of primary resistance to ALK-TKIs for $A L K+$ NSCLC patients. ${ }^{3,4}$ The G1202R mutation is reported to be one of the common resistance mechanisms to firstand second-generation ALK inhibitors (crizotinib, alectinib, and ceritinib). ${ }^{16}$ Interestingly, brigatinib is a highly potent and selective ALK inhibitor, and it maintained substantial activity against all 17 secondary ALK mutants tested in cellular assays and exhibited a superior inhibitory profile compared with crizotinib, ceritinib, and alectinib at clinically achievable concentrations. It has been reported to have potent effects on the refractory G1202R mutant in vitro and in vivo. ${ }^{8,17}$ Brigatinib has been demonstrated to produce a 12.8 month PFS and a $64 \%$ intracranial ORR in crizotinib-refractory patients. However, the patient discussed here did not benefit from brigatinib. Based on the patient's NGS results, it is possible that the G1202R mutation caused primary resistance to brigatinib. 


\section{Jun 2017}

- Diagnosed as lung adenocarcinoma with stage IVa

(cT2bN2M1b)

- CT: $3.6 \mathrm{~cm} \times 2.9$ $\mathrm{cm}$ mass in the upper left lung with lymph node

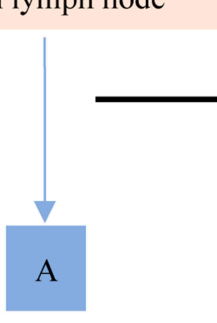

\section{Sep 2017}

- Primary lung lesion stable but lymphatic metastases (LM) emerge
Apr 2018

- CT:lung primary progressd

May 2018

- MR: new brian metastases(BM)

\section{Best OR: SD \\ PFS1: 4 m}

Docetaxel plus cisplatin chemotherapy

\section{Best OR: PR}

PFS2: 8 m

- Crizotinib

Jul 2018

- Primary lung lesion reduced but new mediastinal lymph nod and hydrothorax metastases

\section{Sep 2018}

- Worsend lung symptoms

- Death

Overall survival: $16 \mathrm{~m}$

\section{A. Baseline (Jun 2017)}
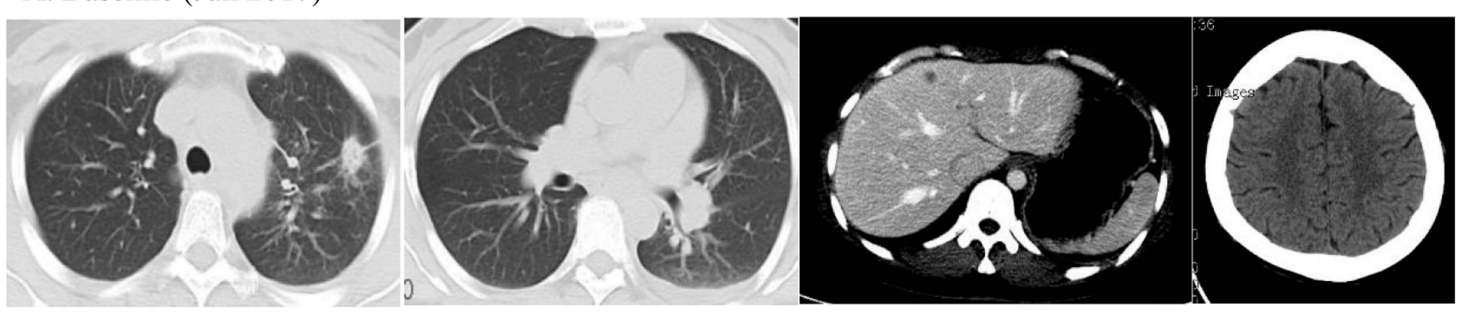

B. PD at 8 m crizotinib (Apr 2018) due to brain metastases (May 2018)
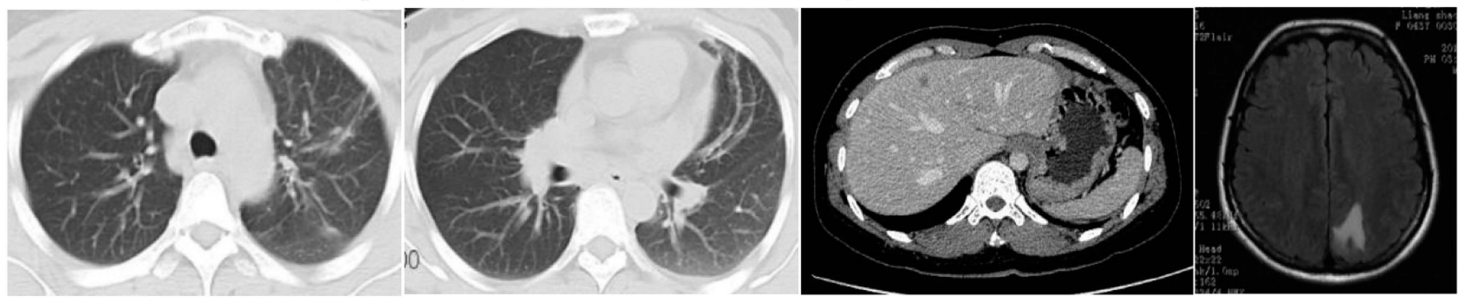

\section{PD after $1.7 \mathrm{~m}$ of brigatinib (Jul 2018)}
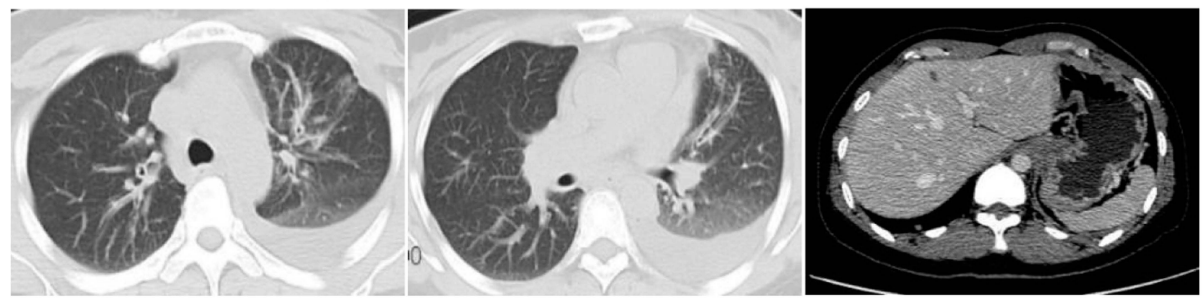

Figure I An illustrated summary of the treatment regimen received by the patient including investigator-assessed objective responses (OR) based on Response Evaluation Criteria in Solid Tumors (RECIST) v.I.I, progression-free survival (PFS) (expressed in months [m]) from each line of treatment. Thoracic computed tomography (CT) at (A) baseline revealed the $3.6 \mathrm{~cm} \times 2.9 \mathrm{~cm}$ mass in the left lung, with lymph node and hepatic segment metastasis, no brain metastases were found. (B) At evaluation of progress response (PD) after 8 month of crizotinib and new brain metastases revealed. (C) At progress response (PD) after 1.7 months of brigatinib.

After brigatinib failure, the patient underwent another NGS testing, and the result showed LIPI-NTRK1 fusion, a new type of NTRK rearrangement, suggesting that this patient developed a potential treatment to NTRK inhibitors. ${ }^{18}$ However, the $A L K \mathrm{G} 1202 \mathrm{R}$ mutation is analogous to resistance mutations that affect the kinase solvent front and will directly interfere with binding with entrectinib and other TKIs with TRK activity. Functional studies have subsequently confirmed that cancer cells harboring these mutations are cross-resistant to all TKIs with anti-TRK activity. As a result, we speculate that both brigatinib and NRTK inhibitors have limited efficacy in this patient due to the G1202R mutation. Meanwhile, given her 


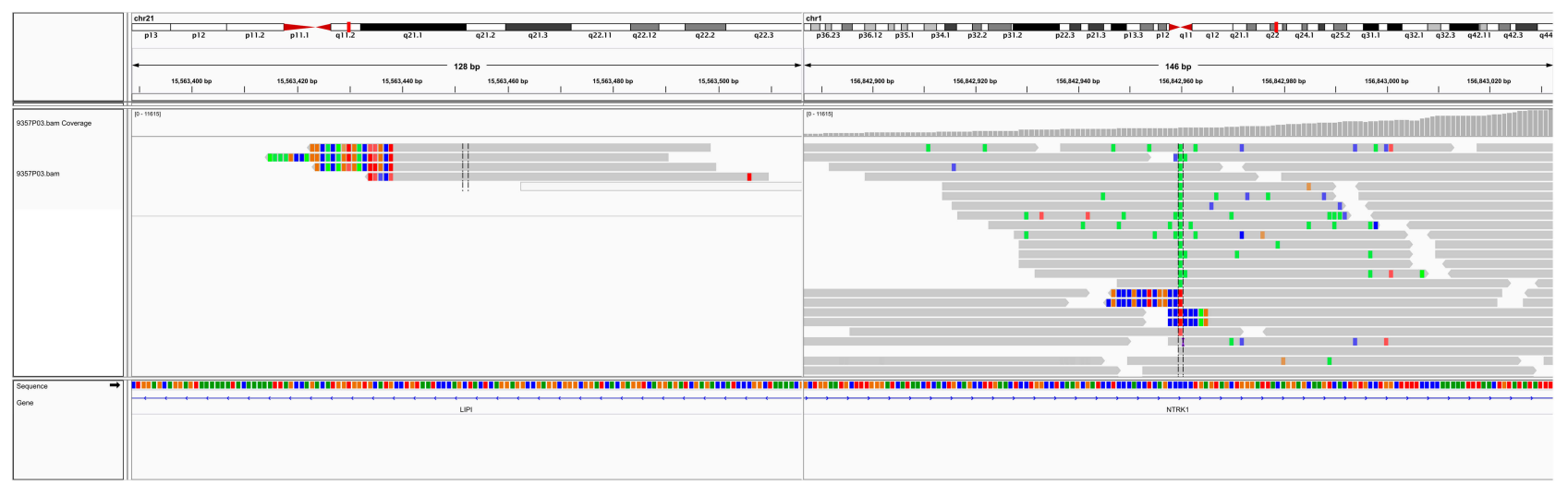

Figure 2 A new fusion form of NTRK rearrangement involving inversion of the LIPI gene (exonsI) and the NTRK gene (exons 8-I7) was identified.

poor physical conditions, switching to an NRTK inhibitor or other targeted drugs is not a viable option for this patient.

In summary, we reported a case of $A L K$-positive NSCLC with primary resistance to brigatinib due to the G1202R mutation. This is also the first report of the LIPI-NTRK1 fusion variant. Although verification of the fusion with fluorescent in situ hybridization or immunohistochemistry was not an option for us due to the lack of tissue, our case provided useful information to better understand the primary resistance to ALK-TKIs. In addition, NGS is valuable to explore the comprehensive genetic characteristics and potential drug targets for patients who experience primary resistance to ALK inhibitors.

\section{Abbreviations}

ALK, Anaplastic lymphoma kinase; NSCLC, non-small-cell lung cancer; ALK-A, ALK amplification; PFS, progressionfree survival; NTRK1, neurotrophic tyrosine kinase 1; EBUS-TBNA, endobronchial ultrasound-guided transbronchial needle aspiration; NGS, next generation sequencing; $\mathrm{PR}$, partial response; $\mathrm{PD}$, progression disease.

\section{Ethics and Consent Statement}

This research was approved by the Research Ethics Committee of the First Affiliated Hospital of Guangzhou University of Chinese Medicine, and written informed consent for participate of the clinical details and images was obtained from the patient's husband.

\section{Consent for Publication}

Written informed consent was obtained from the patient's husband for publication of this case report and any accompanying images.

\section{Acknowledgments}

The authors would like to thank the patient for giving consent.

\section{Author Contributions}

Lizhu Lin contributed to the concept and design and interpretation of data. All authors contributed to acquisition, analysis, and interpretation of data. Zhiwei Xiao and Xuewu Huang drafted the article as the co-first authors. All authors contributed equally in drafting and revising the article and gave final approval of the version to be published, and agree to be accountable for all aspects of the work.

\section{Disclosure}

The authors report no conflicts of interest in this work.

\section{References}

1. Shaw AT, Yeap BY, Mino-Kenudson M, et al. Clinical features and outcome of patients with non-small-cell lung cancer who harbor EML4-ALK. J Clin Oncol. 2009;27(26):4247-4253. doi:10.1200/ JCO.2009.22.6993

2. Zito Marino F, Rocco G, Morabito A, et al. A new look at the ALK gene in cancer: copy number gain and amplification. Expert Rev Anticancer Ther. 2016;16(5):493-502. doi:10.1586/14737140.2016. 1162098

3. Solomon BJ, Kim D-W, Wu Y-L, et al. Final overall survival analysis from a study comparing first-line crizotinib versus chemotherapy in ALK-mutation-positive non-small-cell lung cancer. $J$ Clin Oncol 2018;36(22):2251-2258.

4. Gainor JF, Dardaei L, Yoda S, et al. Molecular mechanisms of resistance to first- and second-generation ALK inhibitors in ALK-Rearranged lung cancer. Cancer Discov. 2016;6(10):1118-1133. doi:10.1158/2159-8290. CD-16-0596

5. Kim DW, Tiseo M, Ahn MJ, et al. Brigatinib in patients with crizotinib-refractory anaplastic lymphoma kinase-positive non-smallcell lung cancer: a randomized, multicenter phase II trial. J Clin Oncol. 2017;35(22):2490-2498. doi:10.1200/JCO.2016.71.5904 
6. Gettinger SN, Bazhenova LA, Langer CJ, et al. Activity and safety of brigatinib in ALK-rearranged non-small-cell lung cancer and other malignancies: a single-arm, open-label, phase 1/2 trial. Lancet Oncol. 2016;17(12):1683-1696. doi:10.1016/S1470-2045(16)30392-8

7. Mezquita L, Planchard D. The role of brigatinib in crizotinib-resistant non-small cell lung cancer. Cancer Manag Res. 2018;10:123-130. doi:10.2147/CMAR.S129963

8. Zhang S, Anjum R, Squillace R, et al. The potent ALK inhibitor brigatinib (AP26113) overcomes mechanisms of resistance to first- and second-generation ALK inhibitors in preclinical models. Clin Cancer Res. 2016;22(22):5527-5538. doi:10.1158/1078-0432.CCR-16-0569

9. Huber RM, Hansen KH, Paz-Ares Rodriguez L, et al. Brigatinib in crizotinib-refractory ALK+ non-small cell lung cancer: 2-year follow-up on systemic and intracranial outcomes in the phase 2 ALTA trial. J Thorac Oncol. 2019.

10. Sharma GG, Cortinovis D, Agustoni F, et al. A compound L1196M/ G1202R ALK mutation in a patient with ALK-positive lung cancer with acquired resistance to brigatinib also confers primary resistance to lorlatinib. J Thorac Oncol. 2019;14(11):e257-e259. doi:10.1016/j. jtho.2019.06.028

11. Farago AF, Le LP, Zheng Z, et al. Durable clinical response to entrectinib in NTRK1-Rearranged non-small cell lung cancer J Thorac Oncol. 2015;10(12):1670-1674. doi:10.1097/01.JTO.00004 73485.38553.f0
12. Vaishnavi A, Le AT, Doebele RC. TRKing down an old oncogene in a new era of targeted therapy. Cancer Discov. 2015;5(1):25-34. doi:10.1158/2159-8290.CD-14-0765

13. Al-Salama ZT, Keam SJ. Entrectinib: first global approval. Drugs. 2019;79(13):1477-1483. doi:10.1007/s40265-019-01177-y

14. Vaishnavi A, Capelletti M, Le AT, et al. Oncogenic and drug-sensitive NTRK1 rearrangements in lung cancer. Nat Med. 2013;19(11):1469-1472. doi:10.1038/nm.3352

15. Farago AF, Taylor MS, Doebele RC, et al. Clinicopathologic features of non-small-cell lung cancer harboring an NTRK gene fusion. JCO Precis Oncol. 2018;2018.

16. Okada K, Araki M, Sakashita T, et al. Prediction of ALK mutations mediating ALK-TKIs resistance and drug re-purposing to overcome the resistance. EBioMedicine. 2019;41:105-119. doi:10.1016/j. ebiom.2019.01.019

17. Zou HY, Friboulet L, Kodack DP, et al. PF-06463922, an ALK/ROS1 Inhibitor, overcomes resistance to first and second generation ALK inhibitors in preclinical models. Cancer Cell. 2015;28(1):70-81. doi:10.1016/j.ccell.2015.05.010

18. O'Reilly EM, Hechtman JF. Tumour response to TRK inhibition in a patient with pancreatic adenocarcinoma harbouring an NTRK gene fusion. Ann Oncol. 2019;30(Suppl 8):viii36-viii40. doi:10.1093/ annonc/mdz385

\section{Publish your work in this journal}

OncoTargets and Therapy is an international, peer-reviewed, open access journal focusing on the pathological basis of all cancers, potential targets for therapy and treatment protocols employed to improve the management of cancer patients. The journal also focuses on the impact of management programs and new therapeutic agents and protocols on patient perspectives such as quality of life, adherence and satisfaction. The manuscript management system is completely online and includes a very quick and fair peer-review system, which is all easy to use. Visit http://www.dovepress.com/ testimonials.php to read real quotes from published authors. 